\title{
MULTIDIRECTIONAL FORCE AND TORQUE SENSOR FOR INSECT FLIGHT RESEARCH
}

\author{
Mansoor Nasir ${ }^{a}$, Michael Dickinson ${ }^{b}$ and Dorian Liepmann ${ }^{a}$ \\ ${ }^{a}$ Dept. of Bioengineering, Berkeley Sensors and Actuator Center, University of California at Berkeley, USA \\ ${ }^{b}$ Bioengineering, California Institute of Technology, Pasadena, CA, USA \\ e-mail: mnasir@socrates.berkeley.edu
}

\begin{abstract}
This paper discusses the development of a multidirectional force sensor for the investigation of the flight dynamic of a tethered fly. The proposed sensor combines the well-understood concepts of piezoresistive force sensing with a unique design that allows for the measurement of forces with more than one degree of freedom (DOF). In addition, the system has been fabricated to support the fly inside a virtual reality arena. The sensor is fabricated on a wafer-level using standard MEMS technology. By directly measuring the thrust, lift, yaw and side slip generated by the fly, complex aerodynamics mechanisms due to rapidly rotating and flapping wings can be better understood.
\end{abstract}

Keywords: Force sensor, Strain gauge, Fruit fly, Flight aerodynamics

\section{INTRODUCTION}

Flying insects have developed sophisticated and unrivalled flight mechanisms permitting them a remarkable range of maneuvers. Fruit flies ( rosphila melanogaster are a model organism for biologists and for insect flight research as within them we find the extremes of organismal design. They represent a superb model system for elucidating general principles that govern complex biological systems and these principles are critical in the realization of any biomemtics inspired flying microrobotic systems.

Flying insects incorporate unsteady aerodynamic principles, which are highly complex due to dynamic nature of forces in both time and spatial domains. Until now the most important tool for understanding flight mechanisms has been high speed videography using a wing beat analyzer from which wing beat frequency and amplitude are deduced [1]. Yaw force measurements were made by optical tracking tethered fruit flies inside a virtual reality arena [2]. However, apart from the elaborate setups required for such experiments, it is difficult to get enough information using such methods to elucidate fundamental issues like the number of independent controls and the coupling between different orthogonal and rotational forces in flying insects. Measuring these forces directly is, therefore, critical to understanding different flight maneuvers and the neural, sensory and mechanical feedbacks that affect the flight motor control.

MEMS technology combines integrated-circuit microfabrication techniques with macroscopic instrumentation principles, makes it possible to develop highly sensitive microsensor systems that are adapted to the requirements of biomimetic studies.

There are many different ways used to measure small forces including, but not limited to pieozoresistive, capacitive, optical and piezoelectric sensing, with each having its advantages and drawbacks. Generally multidirectional force sensing is difficult to do using optical and piezoelectric methods both from design and fabrication point of view. Capacitive force sensing has the advantage of being highly sensitive but multiaxis combdrives have to be used to measure multi DOF forces, again requiring complex fabrication methods. In addition, the requirement for having a physical tether for the fly poses significant problems in all practical designs. Nevertheless, capacitive sensing methods have been used to measure the forces with one DOF (lift) generated by tethered fruit flies with high accuracy [3].

The proposed sensor uses piezoresistive sensing techniques to measure the flight force with greater than single DOF. Piezoresistivity is a material property due to which resistance of the material changes when it is under stress. Sensing techniques using this material property usually incorporate strain gauges located at high stress areas of the structures. Semiconductors strain gauges are up to two orders more sensitive than metal ones and are well-suited for bulk fabrication. Since the underlining theory is well understood and the sensing mechanism is inherently mechanical, novel designs can be used measure multidirectional forces with increased sensitivity.

Strain gauge force sensors have been used extensively in MEMS engineering applications and recently strain gauge sensors have been used in biological applications such as the forces generated by cockroaches while walking and running [4]. Highly sensitivity has also been demonstrated using cantilever style structures [5].

\section{FORCE MICROSENSOR DESIGN}

In developing a microsensor for measurement of flight forces, a number of requirements need to be satisfied. The sensor has to balance the material strength needed to support the weight of the fruit fly while at the same time remain compliant enough to measure forces less than $50 \mathrm{~N}$ [2]. The design should also allow for easy tethering of the fly and placement inside the virtual flight arena [3]. Sensor reusability is important for reliable and repeatable experimental test results.

Fig. 1 shows a solid model of the force sensor that uses piezoresistive strain gauges to measure the lift, thrust and yaw force. 


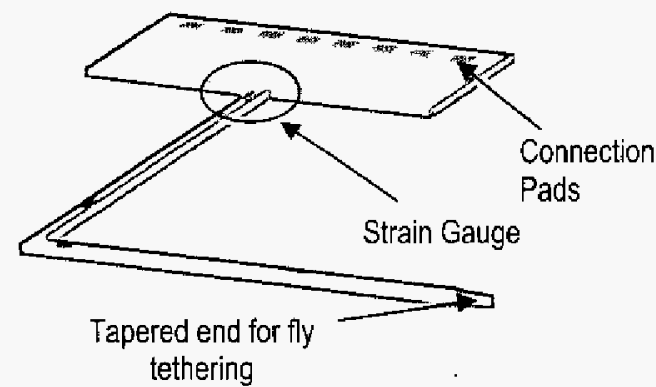

Fig. 1: A model of force microsensor. The fly is tethered at the tapered end of the L-shaped sensor.

The novel design allows replaces the current fly tether and is therefore capable of simultaneously supporting the fly inside the fly arena and measuring the rotational and translational forces. The strain gauges are most frequently used in bridge configurations. The bridge is initially balanced with the strain gauges in the circuitry. When acted upon by a force, the resistance changes and therefore the bridge becomes unbalanced and an output voltage results. Our design has four strain gauges that are used in quarter bridge configuration. The wide base has contact pads to measure the voltage across the strain gauges.

\section{ANALYTICAL MODEL}

Linear beam theory can be used to find the governing equations defining the relation between the forces acting at the tip of the sensor and resulting strain across each strain gauges. The base of the sensor is essentially an anchor and therefore the forces only deform the L-shaped structure of the sensor. The three forces, lift $\left(F_{L}\right)$, Thrust $\left(F_{7}\right)$ and Yaw $\left(M_{y}\right)$ are shown to be acting at the bottom end of the vertical beam. With a free body diagram all the moments resulting from these forces can be visualized (Fig. 2)

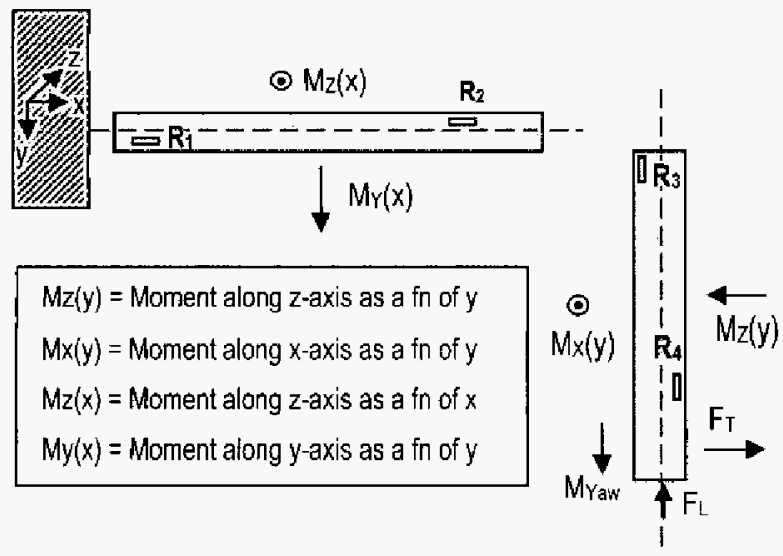

Fig. 2: Free body diagram of the force sensor. The lengths of the vertical and horizontal beams are $L_{V}$ and $L_{H}$ respectively. Right hant rule has been used to show the forces and moments acting on the ends of the beams.
Using a force-moment analysis the four moments shown in Fig. 2 can be written for any point on the horizontal and vertical beams. The relationship between the stress $\sigma$, and moments is given by

$$
s=\frac{M}{I} c \quad \text { where } \quad I=\frac{b h^{3}}{12}
$$

where $c$ is the distance from neutral fiber and $I$ is the moment of inertia Using Hook's law, the strain $\varepsilon$, at the four strain gauge location can related to the moments. These an be expressed as a matrix relation given by

$$
\left(\begin{array}{l}
\varepsilon_{1} \\
\varepsilon_{2} \\
\varepsilon_{3} \\
\varepsilon_{4}
\end{array}\right)=A \cdot\left(\begin{array}{l}
F_{T} \\
F_{L} \\
M_{y a w}
\end{array}\right)
$$

$A$ is a ( $4 \times 3)$ matrix given by

$$
A=\left(\begin{array}{ccc}
\frac{L_{v}}{E I_{1}} c y_{1} & \frac{\left(L_{V}-x_{1}\right)}{E I_{1}} c y_{1} & \frac{1}{I_{2}} c z_{1} \\
\frac{L_{V}}{E I_{1}} c y_{2} & \frac{\left(L_{V}-x_{2}\right)}{E I_{1}} c y_{2} & \frac{1}{I_{2}} c z_{2} \\
\frac{\left(L_{V}-y_{3}\right)}{E I_{3}} c x_{3} & 0 & \frac{1}{G_{T}, \alpha b h_{V^{\prime}}{ }^{2}} \\
\frac{\left(L_{V}-y_{4}\right)}{E I_{3}} c x_{4} & 0 & \frac{1}{G_{T} \cdot \alpha b h_{V}{ }^{2}}
\end{array}\right)
$$

$c x, c y$ and $c z$ are the distances of the four gauges from the center fiber along the $x, y$ and z-axes respectively. The last two terms in the third column are for the torsion in the vertical beam caused due to the yaw force. Values for $E$, the young's modulus and $G_{T}$, the torsional modulus for silicon are given in literature to be around $160 \mathrm{GPa}$ and $80 \mathrm{GPa}[7(\mathrm{a})] . \alpha$ is the coefficient of rectangular bar whose value depends on the ratio of beam thickness and width [7(b)]. The moments of inertias are given by:

$$
\mathrm{I}_{1}=\frac{b h_{H}^{3}}{12} \quad, \quad \mathrm{I}_{2}=\frac{b^{3} h_{H}}{12} \quad, \quad \mathrm{I}_{3}=\frac{b h_{V}^{3}}{12}
$$

where $h_{H}$ and $h_{V}$ are the widths of the horizontal and vertical beams respectively while $b$ is the thickness of the sensor. Finally, the strain can be related to change in electrical resistance by

$$
e=\frac{1}{G} \frac{\Delta R}{R}
$$

where $G$ is the gauge resistance the value of which for Si ranges from 30-150 [6]. The high gauge factor of semiconductors is the main reason why they are so sensitive. A summary of how forces affect the strain gauge resistances is shown in Table 1. 


\begin{tabular}{|c|c|c|c|c|}
\hline & $\mathrm{R}_{1}$ & $\mathrm{R}_{2}$ & $\mathrm{R}_{3}$ & $\mathrm{R}_{4}$ \\
\hline $\mathrm{F}_{\mathrm{T}}$ & $\uparrow$ & $\uparrow$ & $\uparrow$ & $\downarrow$ \\
\hline $\mathrm{F}_{\mathrm{L}}$ & $\uparrow$ & $\downarrow$ & $\leftrightarrow$ & $\leftrightarrow$ \\
\hline $\mathrm{M}_{\mathrm{Y}}$ & $\uparrow$ & $\uparrow$ & $\leftrightarrow$ & $\leftrightarrow$ \\
\hline
\end{tabular}

Table 1: The effect of increasing fores on the resistance of four strain gauges. By simultaneously measuring the change in resistances the forces can be resolved.

\section{MICROFABRICATION}

Bulk micromachining has been used to fabricate the L-shaped sensor out of single crystal silicon wafer and requires only three masks (Fig. 3).
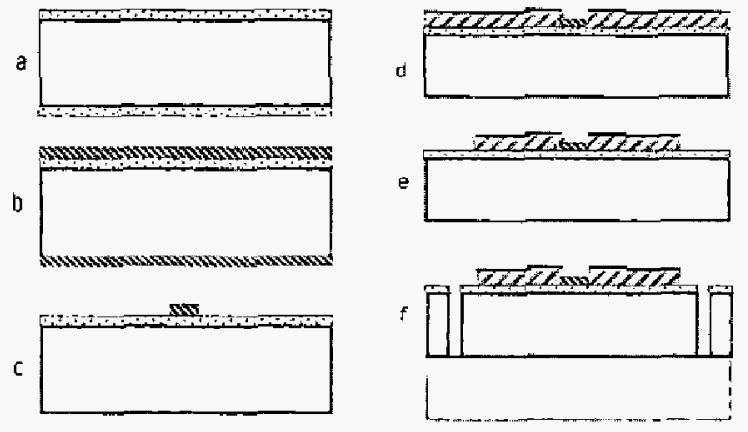

Fig. 3: Microfabrication process (a) A thin oxide layer $(<1 \mathrm{~m})$ is grown on a P-type single crystal Si wafer which will act as a passivation layer. (b) N-doped poly silicon layer ( $400 \AA$ ) is deposited on the wafer after oxide has been removed from the back side of the wafer. The polysilicon deposition step is followed by an annealing step to drive in the dopants. (c) Polysilicon strain gauges are defined using an RIE etch (Mask1). (d) A thick layer of Aluminum (Al) $(1.2 \mathrm{~m}$ ) followed by a very thin layer of Titanium $(<100 \AA)$ is sputtered on the front side of the mask. The Ti layer helps protect the Al leads from getting scratched off. (c) The following lithographic step defines the leads (Mask2). The leads are etched with a combination of dry and wet etches. (f) After a handle bond is attached a last lithographic step (Mask3) is used to RIE etch oxide around the boundaries of the wafer followed by a DRIE through-etch from the front side.

A pair of doped polysilicon strain gauges is microfabricated on each arm of the sensor with Al leads for measuring the voltages across them. There are also four strain gauges on the base of the sensor which are paired up with active strain gauges in the temperaturecompensated quarter bridge configuration. The Al leads are much thicker than the polysilicon layer so that the lead resistances are negligible as compared to the voltage across the strain gauge itself. This also helps to get better step coverage in the overlapping region around the strain gauge and helps reduce the contact resistance. The leads connect to pads that are designed specifically for wirebonding to a printed circuit board (PCB) to which the force sensor is glued (Fig. 4).

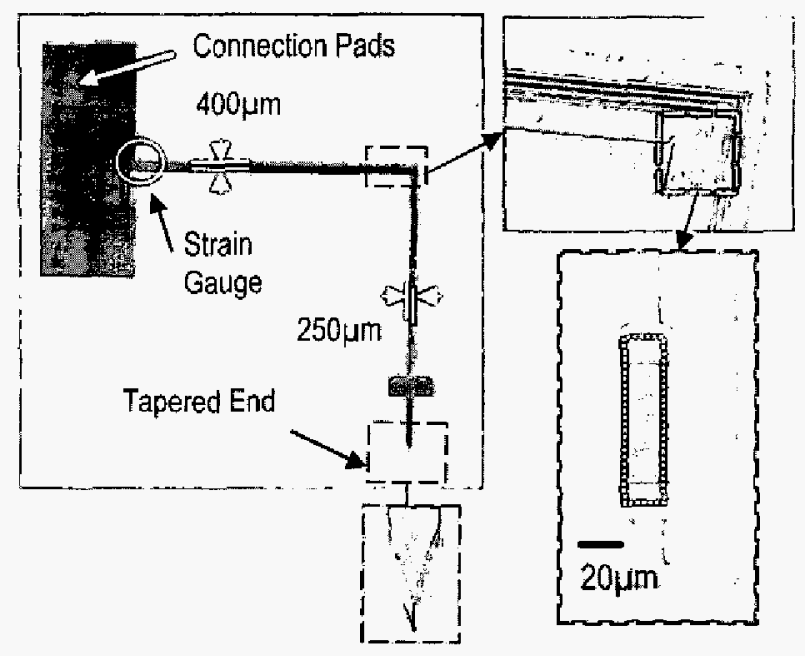

Fig. 4: Microfabricated force sensor with one of the strain gauges highlighted. SEMs of the elbow comer of the force sensor and the tip where the fly is eventually tethered are also shown. The much magnified view shows the Al leads overlapping the piezoresistive n-doped poly strain gauge.

\section{PROTOTYPE SENSOR CALIBRATION}

Initial testing of the sensor with static loads has been done. A force transducer is used to apply a known force at the tip of the sensor after it has been glued and wirebonded to the $\mathrm{PCB}$ and the change in voltage is measured across the four strain gauges while each is held in a quarter bridge configuration. The output voltage of the bridge is amplified using a low noise instrumentation amplifier and high-pass filtered with a cutoff frequency of $2 \mathrm{kHz}$. The whole test setup has to be placed inside a closed hood to prevent the voltage fluctuations due to air drafts. The results for static forces show that the strain gauge voltages vary quite linearly with the applied force (Fig. 5).

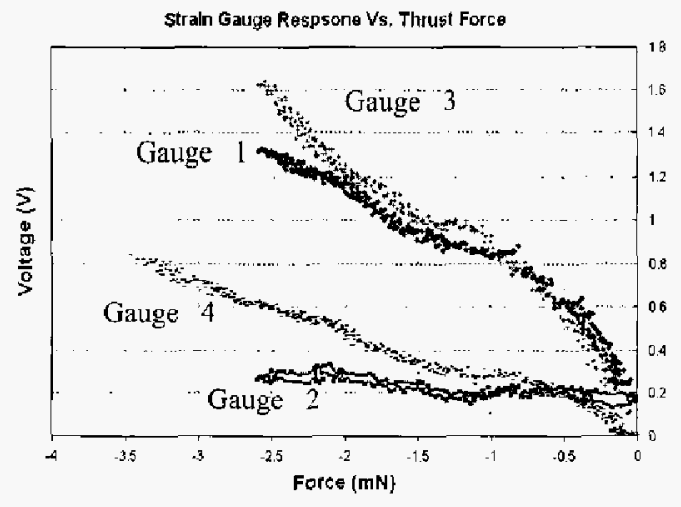

Fig. 5: Change in voltage as a function of applied force shows a relatively linear relationship between the two. The force, thrust in this case produces a different degree of response in the strain gauges depending on their location on the sensor arms. Similar response has been measured for the lift and yaw.

\section{TRANSDUCERS'05}

The 13th Intemational Conference on Solid-State Sensors, Actuators and Microsystems, Seoul, Korea, June 5-9, 2005 
Sensor is also calibrated to for temperature drift (Fig.6).

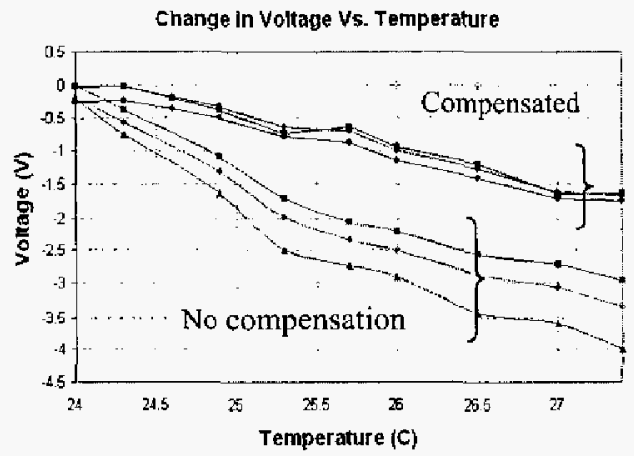

Fig. 6: Voltages from three strain gauges of the same sensor are shown for compensated and uncompensated quarter bridge configuration. The former helps in reducing voltage fluctuations due to change in temperature, However even with the compensated bridge, temperature drift is quite significant.

Forces as small as $100 \mathrm{~N}$ have been successfully measured by hanging small weights at the tip of the sensor. The dynamic response is also under investigation currently and initial results have showed that the fundamental frequencies of all modes are higher than the fly wingbeat frequencies, typically around $200 \mathrm{~Hz}$.

\section{EXPERIMENT AND DISCUSSION}

Initial experiments with tethered fruit flies were done to detect flight forees and also check the feasibility of the experimental setup. Data was collected at $10 \mathrm{kHz}$ (Fig.7). Even though the signal from the fruit flies was observable, the actual forces were too small to be measured with the sensor.

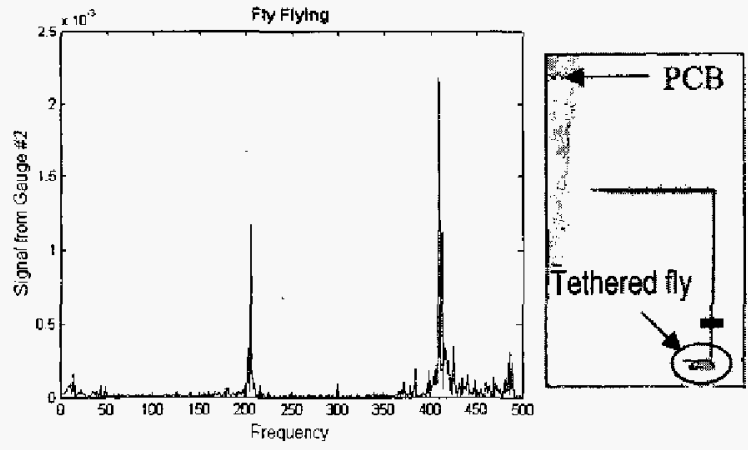

Fig. 7: (Left) FFT of the signal from one of the strain gauges when a fruit fly tethered to the sensor (Right). There is a distinct peak at $200 \mathrm{~Hz}$ which is known to be the wingbeat frequency of the flies. The large peak at $400 \mathrm{~Hz}$ is possibly due to interaction between the first harmonic and the resonant frequency of the bending mode the force sensor.

The calibration data corresponds well with the results of the analytical model and the proof of concept has been verified. However, In order to measure the flight forces applied by the fruit fly the sensor should accurately measure forces smaller then $50 \mathrm{~N}$ with a force resolution of at least $0.1 \mathrm{~N}$ or better. Currently this range of forces is not detectable mainly because of voltage fluctuation caused by temperature changes. Semiconductor strain gauges have a very high thermal coefficient of resistivity because of high thermal conductivity and low thermal expansion coefficient. Design changes are being implemented in which strain gauges are in close proximity and this will insure that the strain gauge pairs of the bridge are isothermal. Increased sensitivity will be accomplished by fabricating the sensors out of thinner wafers and local backside etching of areas where strain gauges are integrated.

\section{CONCLUSION}

A force microsensor system has been developed. The novel design of the sensor allows measurement of forces with greater than single DOF that are being applied by a fly tethered to one end of the sensor. Initial testing of the sensor has verified the proof of concept and forces as small as $100 \mathrm{~N}$ have been successfully measured. The sensor will be used in tethered fly experiments inside a virtual LED arena and by measuring multidirectional forces in real time, complex aerodynamics mechanisms due to rapidly rotating and flapping wings can be better understood.

\section{REFERENCES}

[1] Lehmann, F,-O and Dickinson, M. H. The Changes in Power Requirements and Muscle Efficiency during Eleveated Force Production in the Fruit Fly Drosophila Melanogaster. The Journal of Experimental Biology, 1997, 200, p. 11331143.

[2] Dickinson, M. H. and Lighton, J. R. B. Muscle Efficiency and Elastic Storage in the Flight Motor of Drosophila Science, April $7^{\text {th }} 1995$, Vol. 268, p. 8790.

[3] Potassek DP, Sun Y, Fry SN and Nelson BJ. Characterizing fruit fly flight behavior using a micro force sensor with a new comb drive configuration. J. of MEMS, February 2005, Vol. 14, p 411.

[4] Bartsch, M. S., Partridge, A., Pruitt, B. L., Full. R. J. and Kenny, T. W. A Three-Axis Piezoresistive Micromachined Force Sensor for Studying Cockroach Biomechanics. MEMS, ASME, 2000, Vol. 2, p. 443448.

[5] J.A. Harley and Kenny, T. W. High-sensitivity piezoresistive cantilevers under $1000 \AA$ thick. Applied Physics Letters, Vol. 75, No, 2, pp. 289 291, 1999.

[6] G. T. A. Kovacs, Micromachined Transducers Sourcebook. Boston: WCB McGraw-Hill, 1998 (Chapter 9).

[7] W. C. Young, Roark s Formulas for Stress and Strain, 6th ed., (a) Chapter 8, (b) pg. 243, McGraw-Hill, 1989.

\section{ACKNOWLEDGMENTS}

The researchers would like to thank Packard Foundation for funding this project. 